\title{
Regular and chaotic motion of high altitude satellites
}

\author{
I. Wytrzyszczak ${ }^{\text {a }}$, S. Breiter ${ }^{a}$, W. Borczyk ${ }^{\text {a }}$ \\ ${ }^{a}$ Astronomical Observatory of A. Mickiewicz University, Sloneczna 36, PL 60-286 \\ Poznań, Poland
}

\begin{abstract}
We have computed the integrated autocorrelation function for different families of geosynchronous, inclined orbits in order to detect the regions of chaotic motion. In order to reduce the problems due to high eccentricity orbits, the logarithmic Hamiltonian regularization was applied and a symplectic integrator of the Wisdom-Holman type was implemented. The orbits were integrated for an interval of 10000 days. The results indicate that non-predictable orbits can be found in this relatively short time in the separatrix zone of the $1: 1$ tesseral resonance. Their chaotic nature results from the interchange between libration and circulation type of motion, and from the significant eccentricity growth, caused by the Kozai-Lidov resonance. Some of these orbits intersects the Earth's surface in time shorter than 20 years for a particular initial geometry of interacting bodies.
\end{abstract}

Key words:

Artificial Earth satellites, Chaotic and regular motion, Autocorrelation

PACS: 91.10.Sp, 95.40.+s

\section{Introduction}

Dynamics of geosynchronous, inclined satellites is still an important question, mostly for the safety of geostationary belt objects. The goal of this work is to define regions of the Keplerian elements space where the motion of a geosynchronous satellite under the action of gravitational forces of the Sun, the Moon, the Earth and the solar radiation pressure becomes unpredictable on the timescale of 27 years.

\footnotetext{
* Tel.: +48-61-8292774; fax: +48-61-8292772.

Email address: iwona@amu.edu.pl (I. Wytrzyszczak).
} 
The results of our previous work (Breiter et al., 2005), dedicated to the regularity of geostationary satellites motion problem, indicate that chaotic behavior show such orbits that lie close to the separatrix between the libration and circulation modes, and that this type of chaoticity consist in unforseen changes between these two regimes of motion. Present paper is a continuation of that work. It takes into consideration all geosynchronous orbits with optionally inclination and eccentricity.

From among the Lyapunov exponents, and related or other indicators used to detect the exponential instability of orbits we apply the chaos indicator based on the integrated autocorrelation function.

In previous works, the Lyapunov exponents or related indicators were used to detect the exponential instability of orbits. In the present work we use the chaos indicator based on the integrated autocorrelation function.

We present the maps of this chaos indicator on three different parametric planes: orbital semi-major axes and longitudes of nodes $(a, \lambda)$, eccentricities and arguments of perigee $(e, g)$, and eccentricities and inclinations $(e, i)$. A regularized symplectic integrator helps us to produce such maps for a wide range of initial conditions, including higher initial eccentricities.

\section{Regularized Hamiltonian and the integration scheme}

We studied the motion of a satellite in the geocentric Equator-Equinox reference frame. The perturbing forces were zonal and tesseral harmonics of the Earth's gravity field, gravitational action of the Sun and the Moon and the direct radiation pressure (without a shadow function).

A symplectic, Wisdom-Holman type integrator in the logarithmic Hamiltonian formulation was applied. The method, well suited for highly eccentric orbits, is explained in details in (Mikkola et al., 2002), so here we just recall the Hamiltonian and its partition.

The extended phase space, introduced to formally suppress the explicit dependence of the Hamiltonian on time, consists of Cartesian coordinates, and time variable $(\mathbf{r}, t)$, and their conjugated momenta $\left(\mathbf{p}, p_{0}\right)$. The momentum $p_{0}$ is equal to minus the total energy, i.e. $p_{0}=U-T_{k}$, where $T_{k}$ is the kinetic energy, and $U$ is the force function (negative potential) of the problem

$$
U=\frac{\mu}{r}+U_{1}=\frac{\mu}{r}+U_{E}+U_{M}+U_{S}+U_{S P},
$$

composed of $\mu / r$ - the Keplerian force function of the Earth, $U_{E}$ - it's zonal 
and tesseral part

$$
U_{E}=\frac{\mu}{r} \sum_{l=2}^{N} \sum_{m=0}^{l}\left(\frac{a_{e}}{r}\right)^{l} P_{l m}(\sin \phi)\left(C_{l m} \cos m \lambda_{G}+S_{l m} \sin m \lambda_{G}\right),
$$

where $\phi, \lambda_{G}$ are appropriately the satellite's latitude and longitude in the Greenwich-based rotating frame. In calculus, as the integration is performed in the inertial frame, the longitude $\lambda_{G}$ is replaced with $\lambda_{e q}$, the equinoctical longitude, through relation $\lambda_{G}=\lambda_{e q}-S$, where $S$ is the rotational phase of the Earth computed as $S=n_{E} t+S_{0}$, with $n_{E}$ the angular velocity of Earth's rotation and $S_{0}$ the Greenwich sideral time at the moment $t_{0}$.

The $U_{M}$ and $U_{S}$ are force functions of the Moon and Sun

$$
U_{b}=\mu_{b}\left(\frac{1}{\Delta}-\frac{\mathbf{r}_{\mathbf{b}}}{r_{b}^{3}}-\frac{1}{r_{b}}\right)
$$

with $\Delta=\left\|\mathbf{r}-\mathbf{r}_{\mathbf{b}}\right\|$, and $\mu_{b}$ - the gravity parameter of a third body.

The $U_{S P}$ describes the solar radiation pressure function

$$
U_{S P}=-P_{r} \frac{S}{m} C_{r}\left(\frac{1 \mathrm{AU}}{r_{\odot}}\right)^{2} \frac{\mathbf{r} \cdot \mathbf{r}_{\odot}}{r_{\odot}}
$$

where $P_{r}$ is the solar constant per speed of light, $S / m$ is the satellite area to mass ratio, and $C_{r}$ is the satellite surface reflectivity coefficient.

The logarithmic Hamiltonian (Mikkola et al., 2002)

$$
\mathcal{L}=\ln \left(T+p_{0}\right)-\ln U,
$$

split in two parts

$$
\begin{aligned}
\mathcal{L} & =\mathcal{L}_{k}+\mathcal{L}_{p}, \\
\mathcal{L}_{k} & =\ln \left(T_{k}+p_{0}\right)+\ln r, \\
\mathcal{L}_{p} & =-\ln \left(\mu+r U_{1}\right),
\end{aligned}
$$

permits to formulate the time transformed equations of motion in respect of the new time-type variable $s$.

The quasi-Keplerian motion results from $\mathcal{L}_{k}$ as:

$$
\frac{d \mathbf{r}}{d s}=\frac{\mathbf{p}}{T_{k}+p_{0}}, \quad \frac{d t}{d s}=\frac{1}{T_{k}+p_{0}}=\frac{1}{U}, \quad \frac{d \mathbf{p}}{d s}=-\frac{\mathbf{r}}{r^{2}}, \quad \frac{d p_{0}}{d s}=0,
$$


and the perturbations are generated by $\mathcal{L}_{p}$

$$
\begin{aligned}
\frac{d \mathbf{r}}{d s} & =0, \quad \frac{d t}{d s}=0 \\
\frac{d \mathbf{p}}{d s} & =\frac{1}{\mu+r U_{1}}\left(\frac{\partial\left(r U_{E}\right)}{\partial \mathbf{r}}+\frac{\partial\left(r U_{M}\right)}{\partial \mathbf{r}}+\frac{\partial\left(r U_{S}\right)}{\partial \mathbf{r}}+\frac{\partial\left(r U_{S P}\right)}{\partial \mathbf{r}}\right) \\
\frac{d p_{0}}{d s} & =\frac{1}{\mu+r U_{1}}\left(\frac{\partial\left(r U_{E}\right)}{\partial t}+\frac{\partial\left(r U_{M}\right)}{\partial t}+\frac{\partial\left(r U_{S}\right)}{\partial t}+\frac{\partial\left(r U_{S P}\right)}{\partial t}\right) .
\end{aligned}
$$

The normal set of Hamiltonian equations can be get back via multiplication both sizes of equations (7) and (8) by $d s / d t=U$.

Equations (7) and (8) admit explicit solutions that we composed according to the symmetric, fourth order symplectic integration algorithm of Yoshida (1993). Thanks to the Wisdom-Holman type partition of $\mathcal{L}$ (Wisdom and Holman, 1991), the resulting integrator has the local truncation error proportional to $\varepsilon h^{4}$, where $h$ is the stepsize and $\varepsilon$ is the small parameter proportional to the ratio of $U_{1}$ to $\mu r^{-1}$. Equations (7) can be easily and quickly integrated using the "explicit leapfrog" described by Preto and Tremaine (1999) and Mikkola et al. (2002), whereas the perturbation system (8) admits a simple "kick" solution - a linear function of the integration step.

\section{Autocorrelation algorithm}

Chaos detectors can be divided in two principal categories: ones that measure the exponential divergence of two initially close orbits are more or less directly related with the Lyapunov exponents, other aim at establishing the loss of information about the initial state on a single orbit. The latter group usually exploits the power spectrum of the presumably quasi-periodic motion. It is well known that the autocorrelation of given time series will exponentially decay as a function of lag if the motion is chaotic. This chaoticity test is actually of the spectral type, because the Fourier transform of the autocorrelation is equal to the power spectrum of the original time series (Sprott, 2003). The fastest way to compute the autocorrelation for large number of lags is to use the Fast Fourier Transform algorithm, but its results are less accurate than using a direct approach - especially for larger lags.

There exist two standard definitions of the autocorrelation function, based on constant or instantaneous variances. The constant variance autocorrelation function $C$ at the lag $k$ for an equally spaced time series

$$
r=\left\{r_{i}: \quad r_{i}=r\left(f\left(t_{0}+i h\right)\right), 0 \leq i \leq N\right\}
$$



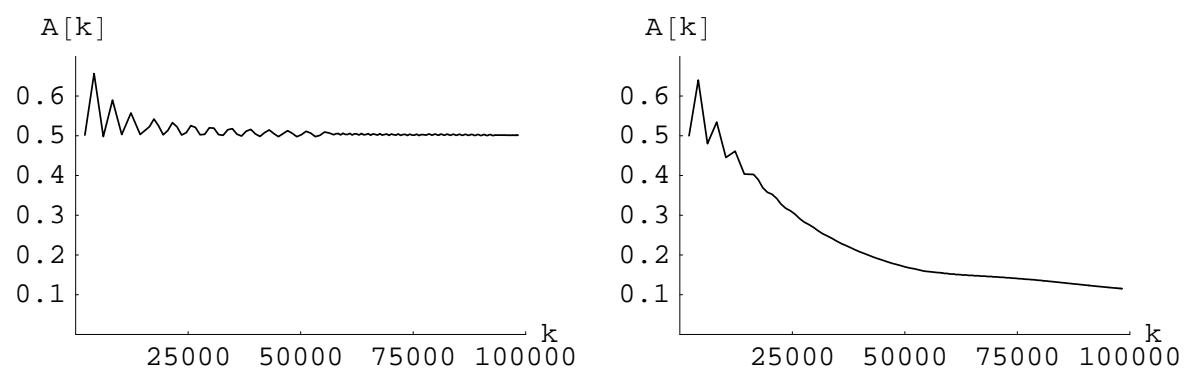

Fig. 1. The integrated autocorrelation function versus the lag number for a regular (left plot) and chaotic (right plot) orbit.

is usually defined by the formula (Sprott, 2003)

$$
C_{k}=\frac{\sum_{i=0}^{N-k}\left(r_{i}-\bar{r}\right)\left(r_{i+k}-\bar{r}\right)}{\sum_{i=0}^{N-k}\left(r_{i}-\bar{r}\right)^{2}},
$$

where $\bar{r}=(N+1)^{-1} \sum_{i=0}^{N} r_{i}$ is a mean value of all $r_{i}$ data.

But a definition more reliable for large lags is known as a product-moment autocorrelation function (Veldman, 1967)

$$
A_{k}=\frac{\sum_{i=0}^{N-k}\left(r_{i}-\bar{r}_{i}\right)\left(r_{i+k}-\bar{r}_{i+k}\right)}{\left(\sum_{i=0}^{N-k}\left(r_{i}-\bar{r}_{i}\right)^{2} \sum_{i=0}^{N-k}\left(r_{i+k}-\bar{r}_{i+k}\right)^{2}\right)^{1 / 2}},
$$

where $\bar{r}_{t}=(t+1)^{-1} \sum_{i=0}^{t} r_{i}$ is the mean value of a $t$-elements subset of data. This definition, although more costly from the computational standpoint, was applied in the present paper.

How to define a single number that reflects the properties of the autocorrelation sequence $A_{k}$ ? For periodic or quasi-periodic orbits $A_{k}$ oscillate between -1 and 1 . In a chaotic system it decays exponentially and time elapsed to the moment when the amplitude of oscillations decays below $1 / e=0.367879$ is proportional to the Lyapunov time (Sprott, 2003). Some authors use the value of the last lag $k$ when the absolute value of the autocorrelation exceeded $1 / e$ as the estimate of the Lyapunov time (Tsiganis et al., 2003). In this paper we use a different indicator: the integrated autocorrelation function, formally defined as the mean of autocorrelation squares $A_{k}^{2}$.

In order to reduce the computation time, not all lags will be used to sum the squares of $A_{k}$. To check for exponential decay it is quite reasonable to sample the large lags more densely than the small ones. Reversing the idea of Wahl et al. (2003), we use exponentially spaced sample points

$$
j_{0}=0, j_{1}=1, j_{k}=j_{k-1}+2^{[(k-1) / B]},
$$


where $[x]$ stands for the integer part of $x$ and $B$ is an integer base number. If $B=1$ the sample points are $\{0,1,2,4,8,16,32, \ldots\}$; for larger values of $B$, the sample points are grouped into sequences of $B$ points, each sequence spaced twice as sparsely as the previous one.

Starting from the series $j_{k}$ we define lags $J_{k}=j_{k^{*}}-j_{k^{*}-k}$, where $k^{*}$ is the last index used to generate sample points according the Equation (11). Hence, $J_{k^{*}}=j_{k^{*}}$ is the last lag considered. The integrated autocorrelation function (IACF) may than be computed with these "reverse exponential lags" using the sum

$$
\mathcal{A}\left(J_{k^{*}}\right)=\frac{1}{J_{k^{*}}}\left(1+\sum_{k=1}^{k^{*}}\left(J_{k}-J_{k-1}\right) A_{J_{k}}^{2}\right) .
$$

All the results presented in this paper are based on the numerical integration covering a time span of $10^{4}$ days with an integration step equal to $1 / 20$ of an orbital period. Autocorrelation was computed from the Cartesian $x$ coordinate of the satellite up to the lags reaching $75 \%$ of the data sample (using $C_{k}$ instead of $A_{k}$ one should typically restrict the lags to some $25 \%$ at most). Comparing the values of the integrated autocorrelation $\mathcal{A}$ obtained with base numbers $B$ equal 20, 40 and 1000, we found that they differ by at most $5 \%$. We have finally adopted $B=40$ as a cautious choice that still guarantee a considerable reduction in computation time.

The integrated autocorrelation function for constant time series $r$ tends asymptotically to $\mathcal{A}=1$. If $r$ represent a uniformly sampled sine wave, the $\mathcal{A}$ function converges to 0.5 . For other periodic and quasi-periodic time series $\mathcal{A}$ tends to a finite value not far from 0.5 . Chaotic orbits result in $\mathcal{A}$ asymptotically decreasing to zero with a speed proportional to the time of exponential decay time. Two exemplary plots of $\mathcal{A}$ as a function of lag for regular and chaotic orbits are presented in Fig. 1.

\section{Results}

The motion of geosynchronous satellites under the joint action of gravity fields of the Earth, the Moon, and the Sun, as well as of the solar radiation pressure, was studied on a time span of $10^{4}$ days (about 27 years), starting from $t_{0}=$ JD2000 $+79^{\mathrm{d}}$. The epoch $t_{0}$ was chosen close the the first day of the Spring of the year 2000, when the Sun stayed close to the Equinox. The geopotential included spherical harmonics up to degree and order 4 of the EGM96 model (Lemoine et al., 1987). Coordinates of the Moon and the Sun, required at each step of integration, were computed according to the "low accuracy formulas" 

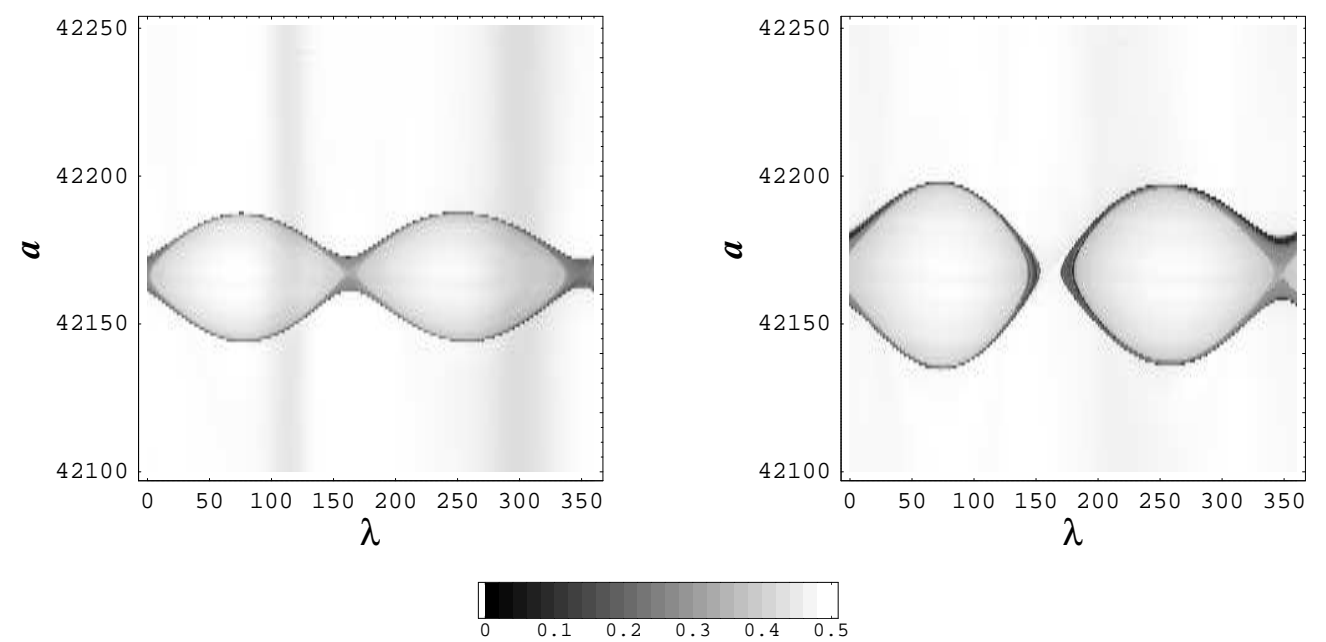

Fig. 2. IACF as a function of the initial $a$ and $\lambda$ for the family of orbits with the initial $g=0^{\circ}$, and $l=0^{\circ}$. Left: the orbits with initial $e=0.05$, and $i=75^{\circ}$. Right: $e=0.2$, and $i=29^{\circ}$.

from the Astronomical Almanac (2000, pp. C1 and D46). In every simulation the area-to-mass ratio of a satellite was taken as $S / m=0.005 \mathrm{~m}^{2} \mathrm{~kg}^{-1}$, and the coefficient of reflectivity of the surface was equal 1.14 for all objects.

\subsection{The semi-major axis - longitude map}

Figure 2 show dependency of the integrated autocorrelation function on initial semi-major axes $a$ and the right ascensions of the ascending node $\lambda$ for two families of inclined orbits. The plot to the left was obtained for the family of orbits with initial equatorial inclination $i=75^{\circ}$ and initial eccentricity $e=0.05$. At the right hand side we present the results for orbits with initial $i=29^{\circ}$ and $e=0.2$. In both cases the remaining initial elements argument of perigee and mean anomaly were $g=l=0^{\circ}$.

The semi-major axis grid is $1 \mathrm{~km}$, starting from $a=42100 \mathrm{~km}$, and the longitude grid is $1^{\circ}$; thus, each map presents a sample of 54000 orbits. Thanks to our choice of the initial epoch $t_{0}$, the right ascension of the node $h$ corresponds to the geographical longitude of a satellite $\lambda$ and was a critical angle of the tesseral resonance $1: 1$.

Similarly to the MEGNO indicator, applied by Breiter et al. (2005) to the problem of regularity of geostationary satellites motion, the autocorrelation function nicely depicts the librational zones of the 1:1 resonance around two stable equilibrium points at longitudes of about $75^{\circ}$ and $255^{\circ}$. The separatrix of the resonance, passing by two unstable points at longitudes $165^{\circ}$ and $345^{\circ}$, contains some chaotic orbits characterized by the value of the IACF in the 


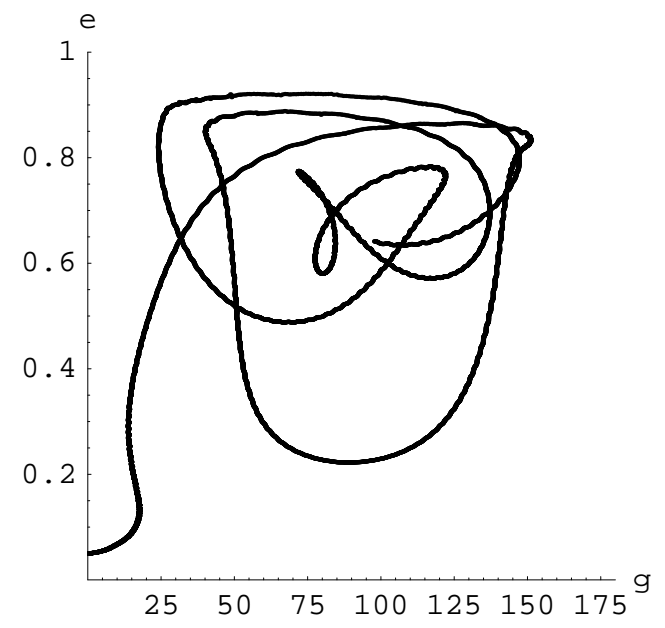

Fig. 3. The eccentricity $e$ and the argument of perigee $g$ for the interval of about 170 years. The remaining initial elements in the rotating equatorial frame were $a=42166.25 \mathrm{~km}, e=0.05, i=75^{\circ}, l=g=0^{\circ}, h=343^{\circ}$.

range $0.09<\mathcal{A}<0.2$. The orbital chaos is reflected in the intermittency of libration and circulation - the phenomenon already observed for the GEO objects (Breiter et al., 2005; Kuznetsov, 2005). Orbits in the separatrix layer may swap between the two libration zones, inner circulation, and outer circulation modes. The motion becomes unpredictable - occasionally even on a time scale of a few years.

There is a clear asymmetry in the number of chaotic orbits in the vicinity of the two unstable points with the excess at the $345^{\circ}$ longitude point. The asymmetry is more visible for orbits with higher eccentricities where the libration zones look like to be disconnected at the unstable point of $165^{\circ}$ longitude. This results from the initial geometry of a satellite, the Moon and the Sun. At the chosen date all three bodies were almost aligned: a satellite and the Moon over the geographical longitude of $165^{\circ}$ and the Sun on the opposite side of the Earth, in the direction to the Equinox coinciding with the Greenwich meridian. Such a configuration causes the Moon to stabilize the motion of a satellite. Individual integrations show that such a motion is regular for longer than 150 years. The "window" of stable satellite motion lasts of about 10 days.

The widths of the two libration zones are also different: the zone surrounding the stable point at longitude $75^{\circ}$ longitude is wider. Both are the effect of higher degree harmonics of the geopotential that differentiate the radial distance of equilibrium points.

The width of the resonant zone diminishes with increasing inclination. For a GEO-belt object it is of the order of $80 \mathrm{~km}$, for a satellite with $i=29^{\circ}$, it does not exceed $60 \mathrm{~km}$, and for $i=75^{\circ}$ it shrinks to about $40 \mathrm{~km}$. 
Outside the separatrix layer, the dynamics looks regular on the interval of 27 years. The conclusion is drawn however on the basis of low initial eccentricities orbits and relatively short integration intervals.

\subsection{Eccentricity - argument of perigee}

Unlike the GEO-belt objects, all satellites inclined to the ecliptic by more than the critical angle $i_{e} \approx 40^{\circ}$ experience a considerable growth of eccentricity. The phenomenon was discovered for artificial satellites by Lidov (1961) and soon rediscovered in the dynamics of asteroids by Kozai (1962). A typical feature of the Kozai-Lidov resonance is the libration of the argument of perigee around $90^{\circ}$ or $270^{\circ}$.

Figure 3 presents dependence of the eccentricity on the argument of perigee for the orbit with high inclination to the equator $\left(i=75^{\circ}\right)$. Its initial eccentricity is equal to $e=0.05$, and at $t_{0}$, when the satellite crossed the equator in its perigee, the longitude of orbital node was equal to $h=343^{\circ}$. The orbit was integrated for a time interval of 63000 days. During the first 50 years, the eccentricity grows from the initial value $e=0.05$ up to $e=0.8$. In the same time, the argument of perigee increases from $0^{\circ}$ to $90^{\circ}$ and then starts to loop around $g=90^{\circ}$. Such a librational motion (for high inclined orbits) around $g=90^{\circ}$ or $g=270^{\circ}$, accompanied by significant growth of orbital eccentricity neither depends on initial eccentricity nor on initial argument of perigee. Even originally circular orbits evolve to very high values of eccentricity, except that they need more time to achieve first maximum. This example sheds some light on the results presented in the next figures.

Figure 4 shows the IACF maps on the plane of initial eccentricities and arguments of perigees. The first map (left) was computed for orbits with initial inclination to the equator of $i=75^{\circ}$; the second (right) was generated for orbits with $i=29^{\circ}$. The remaining elements of both groups, related to the Equator-Equinox frame, were $a=42166.25 \mathrm{~km}, l=h=0^{\circ}$ (note that in this case the initial $g=\lambda$ ). Each orbit was evaluated for the usual $10^{4}$ days interval. Bright vertical regions originating at the bottom lines of the two maps refer to the orbits trapped in the 1:1 tesseral resonance libration around $\lambda=75^{\circ}$ and $\lambda=255^{\circ}$. Darker, vertical "pillars" mark the neighborhood of unstable equilibria. Interestingly, the $g=160^{\circ}$ pattern is more prominent for low inclination orbits. The bright regions in Fig. 4 , with $\mathcal{A}$ very close to 0.5 , consist of stable, librating orbits similar to the one presented at the left hand side of Fig. 5. The initial elements in the rotating equatorial frame for this orbit were: $a=42166.25 \mathrm{~km}, e=0.4, i=29^{\circ} l=h=0^{\circ}$ and the argument of perigee $g=80^{\circ}$. The semi-major axis during the 27 years oscillated in a small range of about $10 \mathrm{~km}$, and in the $x y$ plane (rotating with the Earth) the orbit 

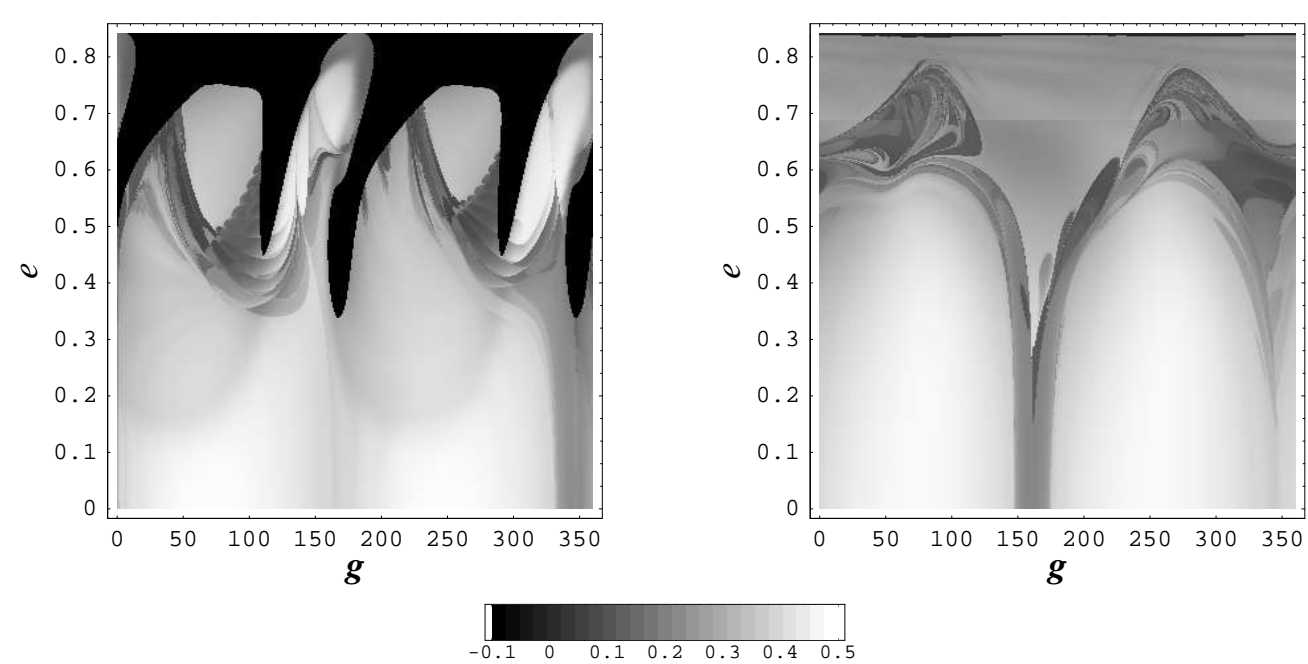

Fig. 4. IACF maps on the eccentricity-perigee plane for the semi-major axis $a=42166.25 \mathrm{~km}$. To the left - a family of orbits with initial $i=75^{\circ}$, to the right - with $i=29^{\circ}$. The remaining initial elements for both groups were $l=h=0^{\circ}$. The value $\mathcal{A}=-0.1$ (black color) was artificially added to the map scale in order to mark that orbits that intersect the Earth surface.

made tight loops around the stable point of $\lambda=75^{\circ}$. Such kind of motion is stable for a very long period of time of the order of hundreds of years. The discussed orbit was separately integrated on the time span of 500 years and during that time its eccentricity oscillated in the range $0.36<e<0.44$, its inclination did not leave the interval $12.5^{\circ}<i<30^{\circ}$, and the semi-major axis showed a small, very long period drift causing the oscillations to change from the range $42162<a<42172 \mathrm{~km}$ in 27 years up to $42156<a<42185 \mathrm{~km}$ after 500 years, generating a bit wider belt of libration in the $x y$ plane.

Darker belts, originating at $e=0, g=160^{\circ}$ or $e=0, g=350^{\circ}$ contain orbits that cross the tesseral resonance separatrix layer. Those at large eccentricities are not subjected to the effects of the 1:1 tesseral resonance, and there the main perturbing factor is the gravitational influence of the Sun and the Moon. Orbits close to the separatrix wander between different regimes of motion like the example given at the right hand side of Fig. 5. The initial elements of the orbit were $a=42166.25 \mathrm{~km}, \mathrm{e}=0.4, i=29^{\circ}, l=h=0^{\circ}$, and $g=180^{\circ}$. During the first 10 years the satellite performed the inner circulation; then it started to swap between the libration, outer circulation and inner circulation in an irregular manner.

The light, stable zones in Fig. 4 for $i=29^{\circ}$ shrink as the eccentricity grows, reflecting the narrowing of the libration zones. But for $i=75^{\circ}$ the situation is different: before the shrinking can be noticed, the Kozai-Lidov resonance comes into play and small stable zones appear, concentrated around $g=90^{\circ}$ and $g=270^{\circ}$. 

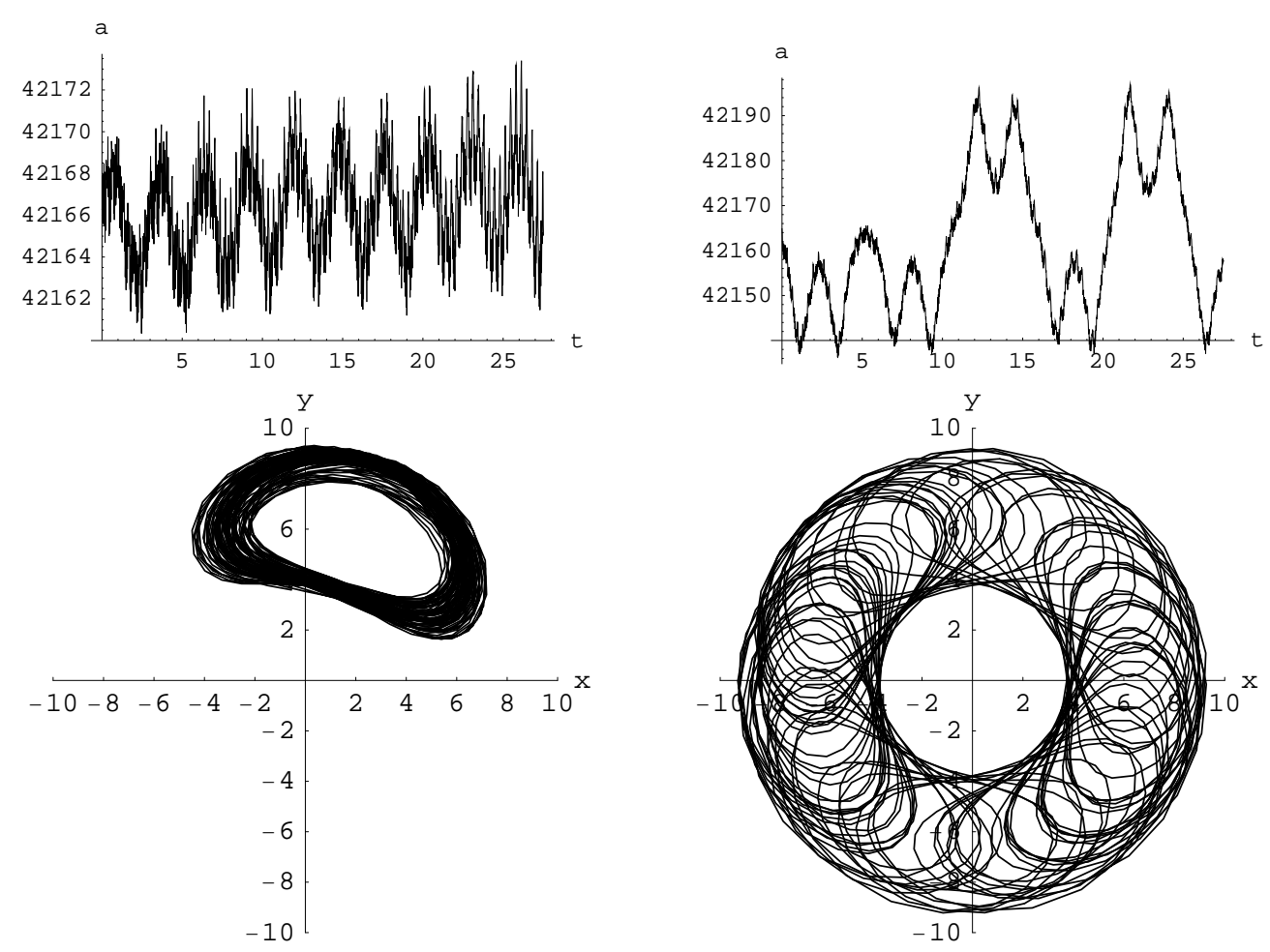

Fig. 5. Two $29^{\circ}$ inclined orbits with elements in the rotating equatorial frame equal: $a=42166.25 \mathrm{~km}, e=0.4, l=h=0^{\circ}$. To the left - the orbit with the initial perigee $g=80^{\circ}$; to the right - with $g=180^{\circ}$. The $x$ and $y$ coordinates are also in the rotating frame; the units are Earth's radii.

It is important to notice a fast eccentricity growth for the orbits with $i=75^{\circ}$ and the initial arguments of perigees close to $0^{\circ}, 90^{\circ}, 180^{\circ}$ or $270^{\circ}$. These zones are marked black on the left map in Fig. 4. Actually they are slightly displaced and concentrate around $g=-10^{\circ}, 110^{\circ}, 160^{\circ}, 290^{\circ}$. The displacement results from the location of the Sun and the Moon at epoch $t_{0}$. At that epoch the Sun and the Moon were almost on opposite sides. The Sun was passing through the vernal equinox whereas the right ascension of the Moon was somewhat larger than $12^{\mathrm{h}}$.

The black zones in Fig. 4 have particular meaning: they are labeled as $\mathcal{A}=$ -0.1 , but this value is purely fictitious - it simply marks the orbits that crossed the Earth surface during the integration interval. For the given semimajor axis it means that their eccentricity exceeded the limit $e=0.84$. The Earth-grazing orbit zones are well confined, consisting of two similar patterns shifted by $180^{\circ}$. They start at $e=0.34$ and $g=170^{\circ}$ or $g=350^{\circ}$. 

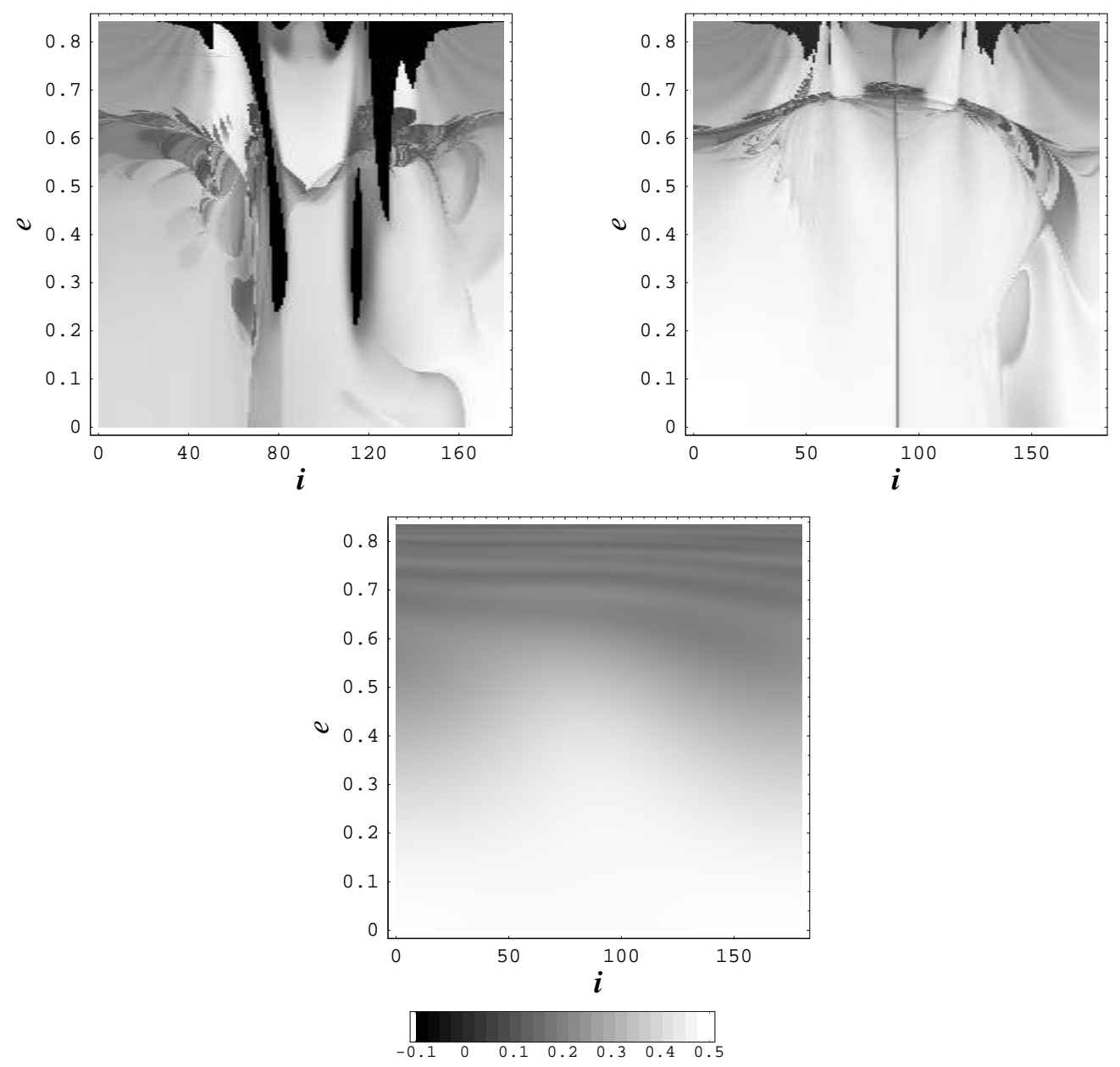

Fig. 6. IACF maps on the eccentricity - inclination plane. Two maps at the top were generated for orbits with $a=42166.25 \mathrm{~km}, l=g=0^{\circ}$, and differ by the value of $h$. The map on the left was made for $h=0^{\circ}$, and to the right with $h=90^{\circ} . \mathcal{A}=-0.1$ marks the Earth-grazing orbits. The map at the bottom presents $a=42466 \mathrm{~km}$, and $l=g=h=0^{\circ}$.

\subsection{Eccentricity - inclination}

Three maps of $\mathcal{A}$ on the eccentricity - inclination plane are presented in Fig. 6. Two of them were computed for the geostationary semi-major axis $a=42166.25 \mathrm{~km}, l=g=0^{\circ}$, and two different right ascensions of the ascending nodes: $h=0^{\circ}$, and $h=90^{\circ}$. The third map presents higher orbits, with the initial semi-major axis equal to $a=42466 \mathrm{~km}$, and $l=g=h=0^{\circ}$.

The map for the supergeostationary semi-major axis shows the $e-i$ space with the third body effects causing the Kozai-Lidov-driven eccentricity growth. However, even for sufficiently high initial inclinations and eccentricities, the time span of 27 years is too short for orbital eccentricity to grow above $e=0.84$, thus the map mostly contains regular orbits. 
Two maps computed for the geostationary semi-major axis show the interplay of the tesseral $1: 1$ and the Kozai-Lidov resonances. The first (left) map was made for initial angles $l=g=h=0^{\circ}$. Recalling that the choice of $t_{0}$ provided us with the vernal equinox close to the Greenwich meridian, we note that the initial right ascension of the node $h$ is also close to the initial longitude of the node. Thus, the initial node of the first family of orbits is near $\lambda \approx 0^{\circ}$, close to the unstable equilibrium point $\lambda=-15^{\circ}$, and was almost aligned with the line connecting Sun and Moon. The whole family of orbits is thus located in the vicinity of the separatrix of the $1: 1$ resonance. The location of third bodies speeds up the eccentricity growth of high inclination orbits, causing the objects starting at $i \approx 80^{\circ}$ or $i \approx 120^{\circ}$ to collide with the Earth. These orbits are colored in black in Fig. 6 .

The stable band up to large eccentricities for $80^{\circ}<i<110^{\circ}$ contains orbits with very slowly precessing line of nodes ( of a hundred up to hundreds of years) and line of apsis (tens to a hundred years).

The picture at the right hand side in Fig. 6 is different, because there are no Earth-grazing orbits with small initial eccentricities. In this case the right ascension of the node $h \approx \lambda=90^{\circ}$, is not far from the stable equilibrium point $\lambda=75^{\circ}$. Moreover, the geometry is such that the line Sun-Moon crosses the line of nodes of each orbit almost perpendicularly. The motion of the whole population is much more regular; only polar orbits suffer higher perturbation resulting in a faster eccentricity growth and in autocorrelation function values in the range of $0.1<\mathcal{A}<0.24$.

The stable band up to large eccentricities for $80^{\circ}<i<110^{\circ}$

\subsection{Origin of chaotic orbits at the separatrix}

As already mentioned in Breiter et al. (2005) chaoticity at the separatix results from the interactions between the tesseral 1:1 resonance and perturbations generated by the Moon and the Sun. We would like now to consider this problem in detail in order to show the zones where one of these perturbations predominates.

The geopotential tesseral terms with coefficients $C_{2,2}$ and $S_{2,2}$ cause librations of the semi-major axis with a period of about 3 years. These terms give the leading contribution to the evolution of orbits in the neighborhood of the stable libration points. Higher order resonances, the Sun and the Moon do not seriously influence the motion in this zone. One example of such orbits is plotted in the Fig. 7a. This is the orbit with the initial semi-major axis equal to $a=42166.25 \mathrm{~km}$, the eccentricity $e=0.4$, the inclination $i=29^{\circ}$, the argument of perigee $g=100^{\circ}$, and remaining Keplerian angles $l=h=0^{\circ}$. 
a) $a=42166.25 \mathrm{~km}, e=0.4, i=29^{\circ}, g=100^{\circ}, l=h=0^{\circ}$
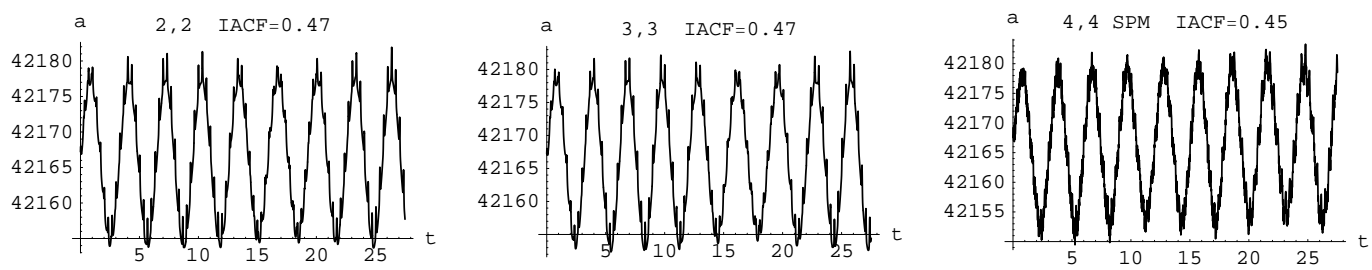

b) $\quad a=42176 \mathrm{~km}, e=0.001, i=29^{\circ}, l=g=h=0^{\circ}$


c) $\quad a=42190 \mathrm{~km}, e=0.2, i=29^{\circ}, h=30^{\circ}, l=g=0^{\circ}$


d) $a=42190.5 \mathrm{~km}, e=0.2, i=29^{\circ}, h=30^{\circ}, l=g=0^{\circ}$
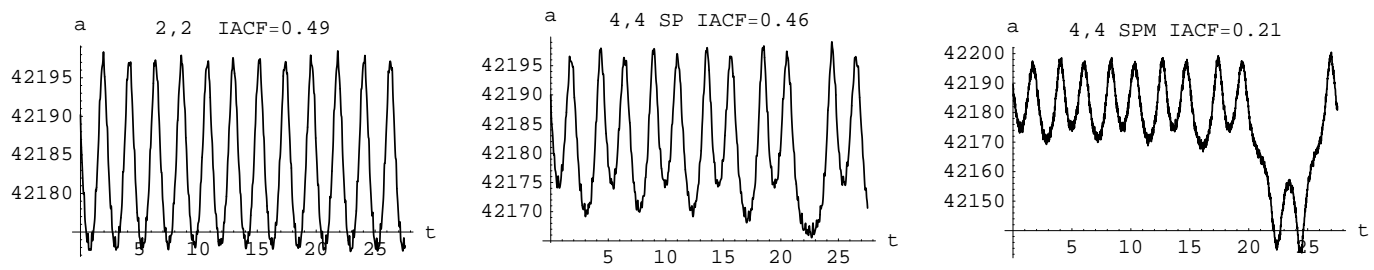

e) $\quad a=42166.25 \mathrm{~km}, e=0.65, i=29^{\circ}, g=100^{\circ}, l=h=0^{\circ}$
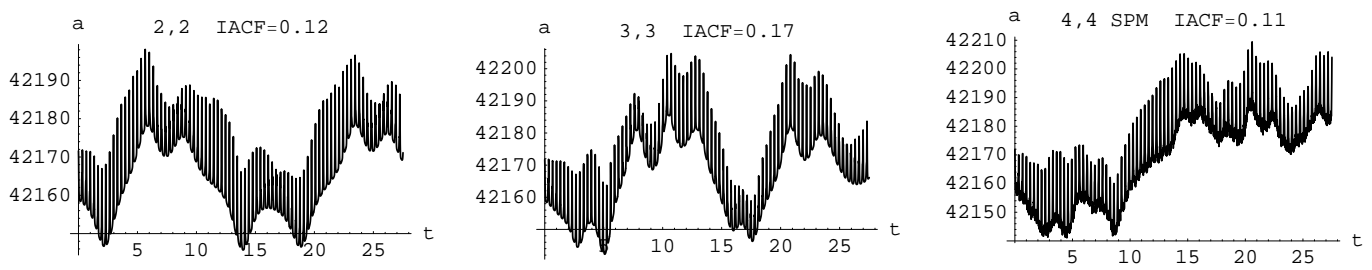

Fig. 7. Semi-major axis of four different orbits versus time. The perturbing forces are labeled at the top of each plot. Numbers stands for the degree and order of geopotential terms. S marks Sun's, M - Moon's and P - solar radiation pressure perturbations. 
The initial geometry is such that the argument of perigee is very close to the resonant angle such that the plot presents librations around the stable point at $\lambda=75^{\circ}$. This kind of a stable librational motion occurs for orbits with the initial eccentricities less than $e=0.6$.

Orbits at the separatrix present a diversity of the influence of individual forces. There are some for which the main perturbing factor are the overtones of the $1: 1$ tesseral resonance. Figure $7 \mathrm{~b}$ presents the orbit with the initial semi-major axis $a=42176 \mathrm{~km}, e=0.001, i=29^{\circ}$, and $l=g=h=0^{\circ}$. The evolution of its semi-major axis is defined by the third sectorial harmonics of the geopotential $C_{3,3}$ and $S_{3,3}$. There also exist orbits for which the leading perturbing term is the second overtone of the resonance, i.e. the fourth sectorial harmonic. In both cases the Moon and Sun have no important influence. This type of orbits mainly exist for small eccentricities, usually less than $e=0.2$.

The evolution of the semi-major axis of orbits with higher eccentricities $0.1<$ $e<0.6$ is governed mainly by the lunisolar perturbations. Interestingly, the influence of the Moon and the Sun can stabilize the motion in some cases, but usually it provokes changes of the regime of motion. In Fig. 7c, d two orbits with their semi-major axes differing only by $0.5 \mathrm{~km}$ are presented. The main cause of irregularity evolution of the semi-major axis for the orbit in Fig. 7c is the Sun that lowers the value of the integrated autocorrelation function to $\mathcal{A}=0.12$. The Moon smoothes the curve, stabilizes the motion, and increases $\mathcal{A}$ to 0.15 . The orbit with the semi-major axis higher by $0.5 \mathrm{~km}$, plotted in Fig. 7 d, presents a counterexample: there the Moon destabilizes librational motion.

Orbits with eccentricities in the range $0.6<e<0.75$, especially those in the dark gray regions of the Fig. 4 (right), are specific: the second tesseral harmonics alone already cause the changes from the libration regime to the inner or outer circulation regimes of motion. Other perturbing factors (higher tesseral harmonics, the gravitational influence of the Moon or the Sun) additionally influence the motion. An example of such an orbit is plotted in Fig. 7e.

\section{Conclusions}

The integrated autocorrelation function has proved to be a suitable to identify different types of orbits. Similarly to the GEO belt simulations results, we found chaotic geosynchronous orbits on the separatrix layer of the 1:1 tesseral resonance that results from the interaction with lunisolar perturbations. The chaos manifests itself through the phenomenon of intermittency between libration and circulation regimes, already known for geostationary orbits. Another source of chaotic or even Earth-grazing orbits is the interaction of the 1:1 
tesseral resonance with the Kozai-Lidov resonance. The latter generates a significant growth of the eccentricity and provokes a broadening of the width of the 1:1 tesseral separatrix, especially for orbits with initial eccentricities higher than $e=0.2$. There exist some groups of orbits that increase the eccentricity to the value that the objects intersect the Earth's surface in 27 years or less. The geometry of mutual initial locations: Sun - Moon - satellite plays a key role in this phenomenon and demands further research. The super-GEO space, during the investigated period of 27 years, looks regular and quasi-periodic.

\section{Acknowledgements}

The authors thank both referees for their helpful assistance. The research was partially supported by the Polish State Committee of Scientific Research (KBN) grant 5T12D 02623 and the Spanish Ministry of Science and Technology, project \# ESP2005-07107.

\section{References}

—, 2000. The Astronomical Almanac. Nautical Almanac Office, Washington and London.

Breiter, S., Wytrzyszczak, I., Melendo, B., 2005. Long-term predictability of orbits around the geosynchronous altitude. Advances in Space Research, 35, 1313-1317.

Kozai, Y., 1962. Secular Perturbations of Asteroids with High Inclination and Eccentricity. The Astronomical Journal, 67/9, 591-598.

Kuznetzov, E.D., 2005. Stochastic dynamics of geosynchronous satellites. Celestial Mechanics and Dynamical Astronomy, submitted.

Lemoine, F. G., Kenyon, S. C., Factor, J. K., Trimmer, R., Pavlis, N. K., Chinn, D. S., Cox, C. M., Klosko, S. M., Luthcke, S. B., Torrence, M. H., Wang, Y. M., Williamson, R. G., Pavlis, E. C., Rapp, R. H., Olson, T. R., July 1987. The development of the joint NASA, GSFC and NIMA geopotential model EGM96. Tech. Rep. NASA/TP-1998-206861, NASA Goddard Space Flight Center, Greenbelt, Maryland, 20771 USA.

Lidov, M.L., 1961. Evolution of artificial planetary satellites under the action of gravitational perturbations due to external bodies. Iskusstviennye Sputniki Zemli (in Russian) 8, 5-45.

Mikkola, S., Palmer, P., Hashida, Y., 2002. An implementation of the logarithmic Hamiltonian method for artificial satellite orbit determination. Celestial Mechanics and Dynamical Astronomy, 82, 391-411.

Preto, M., Tremaine, S., 1999. A class of symplectic integrators with adaptive 
time step for separable Hamiltonian systems. The Astronomical Journal, 118, 2532-2541.

Sprott, J.C., 2003. Chaos and Time-Series Analysis. Oxford University Press, New York.

Tsiganis, K., Varvoglis, H., Hadjidemetriou, J.D., 2002. Stable chaos in highorder Jovian resonances. Icarus, 155, 457-474.

Wahl, M., Gregor, I., Patting, G.M., Enderlin, J., 2003. Fast calculation of fluorescence correlation data with asynchronous time-correlated single-photon counting.Optics Express, 11, 3583-3591.

Wisdom, J., Holman, M., 1991. Symplectic maps for the n-body problem. The Astronomical Journal, 102, 1528-1538.

Veldman, D.J., 1967. Fortran programming for the behavioral sciences. Holt, Rinehart and Winston, New York.

Yoshida, H., 1993. Recent progress in the theory and application of symplectic integrators. Celestial Mechanics and Dynamical Astronomy 56, 27-44. 